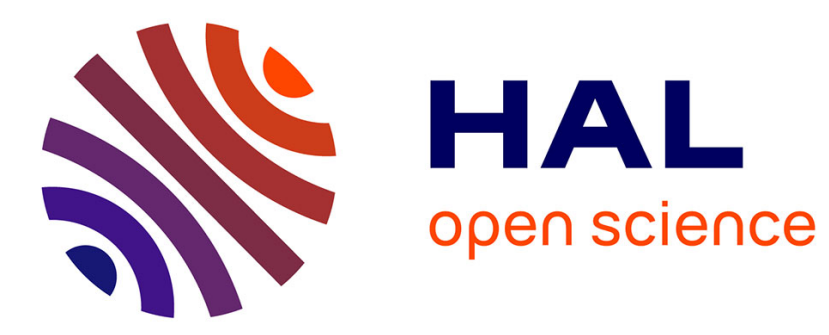

\title{
POUILLON, François, 2017, Bédouins d'Arabie. Structures anthropologiques et mutations contemporaines
}

Catherine Baroin

\section{To cite this version:}

Catherine Baroin. POUILLON, François, 2017, Bédouins d'Arabie. Structures anthropologiques et mutations contemporaines. Journal des Africanistes, 2018, pp.156-159. hal-02337725

\section{HAL Id: hal-02337725 \\ https://hal.parisnanterre.fr/hal-02337725}

Submitted on 29 Oct 2019

HAL is a multi-disciplinary open access archive for the deposit and dissemination of scientific research documents, whether they are published or not. The documents may come from teaching and research institutions in France or abroad, or from public or private research centers.
L'archive ouverte pluridisciplinaire HAL, est destinée au dépôt et à la diffusion de documents scientifiques de niveau recherche, publiés ou non, émanant des établissements d'enseignement et de recherche français ou étrangers, des laboratoires publics ou privés. 


\section{Pouillon, François, 2017, Bédouins d'Arabie. Structures anthropologiques et mutations contemporaines Paris, IISMM/ Karthala, "Terres et gens d'islam », 296 p.}

\section{Catherine Baroin}

Rappelons d'abord que François Pouillon est l'éditeur d'une œuvre scientifique monumentale, le Dictionnaire des orientalistes de langue française, paru chez Karthala en 2008. Revu et complété, ce dictionnaire fut réédité en 2012 pour atteindre 1112 pages ! L'orientalisme érudit de notre auteur s'exprime aussi dans cet ouvrage consacré aux Bédouins d'Arabie. Son érudition se manifeste d'ailleurs dans bon nombre de ses publications précédentes, après de premières enquêtes de terrain en Tunisie saharienne qui firent de lui d'emblée un spécialiste du monde bédouin, et plus largement du pastoralisme nomade ainsi que du monde arabe. Cependant, ce sont des enquêtes menées en Arabie saoudite, en 1979 et 1980, qui apportent à ce livre son éclairage le plus moderne, en l'amenant à souligner les énormes mutations de cette société pastorale dans la période récente.

Les chapitres de l'ouvrage sont très hétérogènes, ce qui lui donne l'allure d'un fourre-tout quelque peu décousu. Pourtant, il découle de l'ensemble un bon éclairage global sur les Bédouins d'hier et d'aujourd'hui, ainsi que de riches réflexions théoriques sur les pasteurs nomades en général. L'intérêt de cette publication touche à trois domaines : l'historiographie, le débat théorique sur le pastoralisme, et les mutations de la société bédouine actuelle.

La composante historiographique de l'ouvrage est très forte. II retrace en effet " l'invention du monde bédouin " dans l'imaginaire occidental, révélé par l'analyse de la littérature depuis le siècle des Lumières (chap. ii et iii), et commente les principaux travaux sur ces pasteurs du désert. Un chapitre enthousiaste (chap. v) est consacré à Charles Doughty. Dans les années 1876-1878, ce voyageur anglais fut I'un des premiers Européens à parcourir l'Arabie. II publia, à la suite de ce voyage, un volumineux ouvrage devenu un grand classique, Travels in Arabia deserta (1888), traduit et publié tardivement en français, en 2002. Pouillon souligne la très grande valeur de cette source d'information sur la société bédouine d'autrefois. Son appréciation est plus mesurée sur Wilfred Thesiger, qui fut le premier explorateur européen à s'aventurer dans le " désert des déserts ", au sud de l'Arabie saoudite, au début du siècle dernier. Relève également de l'historiographie le chapitre iv du livre, consacré au cheval arabe, ainsi que le chapitre ix qui évoque le regard de Jacques Berque sur les Bédouins du Sahara algérien, alors que cet auteur n'a mené lui-même aucune enquête chez ces nomades. Ces divers chapitres mis ensemble constituent une excellente introduction à l'étude des Bédouins. La revue de la littérature qui leur est consacrée est complétée par une précieuse bibliographie en fin de volume. Aucune bibliographie, cependant, n'est exhaustive, et, si large soitelle, on regrette de ne pas y trouver le superbe témoignage d'Antonin Jaussen publié en 1908, Coutumes des Arabes au pays de Moab.

Le volet théorique du livre, pour sa part, se donne à voir dans deux chapitres très différents, et distants l'un de l'autre dans l'ouvrage. Le premier est le chapitre i, intitulé « Une théorie générale du nomadisme pastoral ", tandis que le second n'apparaît qu'en septième place et porte sur la question spécifique du dénombrement du bétail.

Le chapitre i est un commentaire critique de l'ouvrage d'Anatoly Khazanov, Nomads and the Outside World (1984), qui traite du nomadisme pastoral à l'échelle mondiale et soulève d'importantes questions théoriques. Ce livre inspira nombre de questions abordées par Pouillon dans le cadre de son séminaire "Anthropologie des pasteurs nomades", ouvert alors à l'EHESS. Ce sont notamment celle de l'égalitarisme dans les sociétés nomades (qui fut à l'époque abondamment débattue), de leurs liens avec les citadins et les États voisins (à la suite des pertinentes observations de ce précurseur lointain que fut Ibn Khaldûn), la question de l'autonomie économique des nomades, du 
rôle de la prédation, des rapports avec les sédentaires établis dans des oasis ou aux marges de leur domaine. Comme Khazanov, Pouillon insiste en particulier sur le fait que l'exploitation des sédentaires constitue toujours la base économique des nomades, sans laquelle ils ne sauraient survivre (p. 163).

Dans le chapitre vii, il aborde la délicate question du recensement du bétail chez les nomades, en se basant cette fois sur son expérience de trois terrains, chez les Bédouins du Sud tunisien, les Peuls du Sénégal et les Bédouins d'Arabie. II montre comment des situations concrètes très différentes poussèrent les éleveurs à réagir de façon diamétralement opposée. Chez les Bédouins de Tunisie, il note les réticences face à l'enquête sur ce sujet, qui prend l'allure d'une investigation fiscale suscitée par les besoins de statistiques des organismes nationaux et internationaux. Les enquêtes personnelles de l'auteur sur les Bédouins et leurs moutons du Sud tunisien le portent à remarquer que « le bétail, capital symbolique s'il en est, ressortissait de l'ordre du caché (harâm), au même titre que l'intimité des filles du lignage. II ne pouvait pas faire l'objet d'investigations parce qu'il était le bien par excellence et, de ce fait, inestimable » (p. 128). Pour autant, la situation est bien différente en Arabie où une aide aux éleveurs fut apportée par l'État en proportion du nombre des animaux déclarés. II en résulta, comme on pouvait l'escompter, une surestimation des effectifs, les déclarations des éleveurs prenant alors un caractère essentiellement politique. Mais les chèques remis aux nomades ne furent pas honorés, et l'État par la suite mit en place une autre politique d'aide aux nomades, en subventionnant les aliments pour le bétail.

À propos des Peuls du Sahel sénégalais, chez lesquels il eut également l'occasion d'enquêter, Pouillon constate le même refus de dire combien d'animaux on possède que chez les éleveurs tunisiens. Ses remarques sur la conception complexe du troupeau, à l'opposé de celle des vétérinaires, sont particulièrement bienvenues (p. 140-146). "On surveille un troupeau non comme une collection mais comme une structure » (p. 134). Pouillon souligne :

On est ainsi ramené au problème de fond : celui de l'appropriation. Car à qui appartient le troupeau ? À celui (ou à celle) qui le garde ? À celui (ou à celle) qui le trait ? À celui qui peut disposer de ses produits, lait ou croît, temporairement ou définitivement? À celui qui peut décider de sa dévolution en le prêtant, le confiant ou le vendant, ou interdire telle ou telle de ces affectations ? À celui qui est en situation d'en hériter ? À celui qui est en position politique, économique ou militaire d'en soutirer une part ? À celui enfin qui aurait le pouvoir (le droit) de le compter? À tous ceux-là assurément, à condition que l'on précise chaque fois sous quel rapport et à quel degré (p. 146).

Telle est, remarquablement résumée, la complexité des liens au bétail qui s'exercent dans toutes les sociétés pastorales, quelles qu'elles soient, et que toute ethnographie se doit d'investiguer. En effet, ce " problème de fond " apporte un éclairage essentiel sur les relations interpersonnelles, qui dans ces sociétés sont largement fonction de la nature des liens (et des droits) de chacun sur chaque animal du troupeau (car ces droits varient en outre d'un animal à l'autre). C'est un point sur lequel j'ai moi-même été amenée à mettre l'accent, à propos des Toubou.

La dernière partie de l'ouvrage porte sur les mutations contemporaines de la société bédouine d'Arabie, et se base sur des enquêtes menées par l'auteur durant sept mois, entre 1979 et 1980 . II s'agit donc de données déjà anciennes, et la situation décrite a vraisemblablement encore beaucoup changé depuis. Pour autant, les changements dont elles témoignent sont si radicaux qu'on a du mal à y reconnaître la société bédouine d'autrefois. L'auteur souligne la remarquable capacité d'adaptation de ces Bédouins d'Arabie. Ils ont abandonné les longues itinérances d'antan à la recherche de 
pâturages, et transportent désormais leurs animaux en camions pour aller là où la pluie est tombée. Si l'eau y fait défaut pour l'abreuvage, elle est apportée sur place en citerne. Et lorsque le pâturage manque, on achète du fourrage pour nourrir les animaux. Le pastoralisme est devenu une industrie, qui suppose des investissements en camions, en citernes, en fourrage et en essence, et le bas prix du pétrole en Arabie saoudite en est une première condition. De plus, l'élevage camelin peu rentable est largement abandonné au profit de celui des chèvres et des moutons dont le débouché est assuré par les fêtes familiales, le ramadan et, surtout, le pèlerinage à la Mecque. Le gardiennage du bétail, travail d'homme autrefois, est de plus en plus assuré par les femmes, ce qui est un autre important changement dans l'organisation familiale du travail.

Par ailleurs, les Bédouins ont été fortement incités à se sédentariser, et n'ont souvent guère eu d'autre choix. Mais cette option suppose aussi des investissements coûteux, pour creuser un puits et acquérir des terres à irriguer. Ce nouveau mode de vie est une vraie révolution, surtout quand on songe au mépris ancien des nomades pour le travail de la terre. Disposer de la main-d'œuvre nécessaire pose souvent problème, si bien que certains jeunes sont contraints de rester sur place pour apporter leur force de travail à l'entreprise familiale. Par manque de capital, ils sont souvent dans l'impossibilité de se marier, car les potentielles épouses refusent les dures réalités de la vie loin des villes.

Menacée de surcroît par les problèmes récurrents de sécheresse, la survie des Bédouins est précaire. Comme le souligne Pouillon, c'est le maintien d'un très fort système de valeurs qui permet de l'assurer. Celui-ci se caractérise par la vénération pour le modèle patriarcal, l'idéal de fécondité (qui permet de disposer de main-d'œuvre), l'idéal de solidarité des agnats, l'acceptation d'un mode de vie rustique, le sacrifice de la réalisation individuelle au profit du groupe (avec le maintien de l'indivision patrimoniale et le célibat de certains). De plus en plus, le soutien extérieur de parents exilés à l'étranger, ou d'autres partis vivre en ville où ils bénéficient d'un emploi ou d'une autre source de revenu, est la clé du maintien du reste de la famille dans des activités pastorales ou agricoles.

Ainsi, la vie des Bédouins actuels d'Arabie n'a plus aucun rapport avec celle d'autrefois. Le mode de vie nomade est désormais folklorisé pour attirer les touristes. Remercions François Pouillon de nous apporter cette image - un peu triste - d'un monde moderne jusqu'à présent peu documenté, où le pastoralisme dénaturé apparaît comme un vestige du passé. 